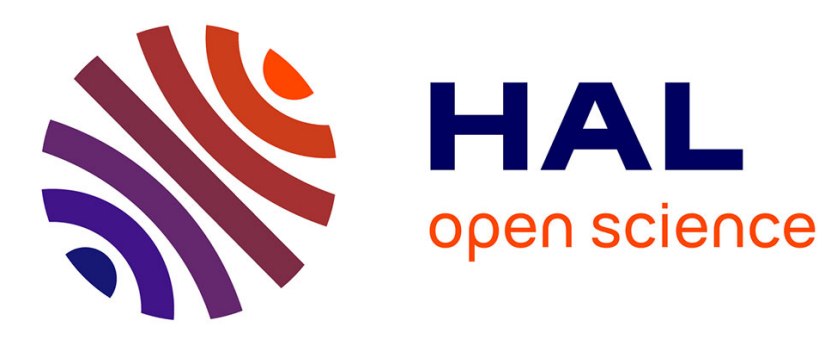

\title{
Visual Servoing/Tracking Using Central Catadioptric Cameras
}

\author{
Joao Barreto, Frederick Martin, Radu Horaud
}

\section{To cite this version:}

Joao Barreto, Frederick Martin, Radu Horaud. Visual Servoing/Tracking Using Central Catadioptric Cameras. International Symposium on Experimental Robotics (ISER '02), Jul 2002, Sant'Angelo d'Ischia, Italy. pp.245-254, 10.1007/3-540-36268-1_21 . inria-00590161

\section{HAL Id: inria-00590161 https://hal.inria.fr/inria-00590161}

Submitted on 3 May 2011

HAL is a multi-disciplinary open access archive for the deposit and dissemination of scientific research documents, whether they are published or not. The documents may come from teaching and research institutions in France or abroad, or from public or private research centers.
L'archive ouverte pluridisciplinaire HAL, est destinée au dépôt et à la diffusion de documents scientifiques de niveau recherche, publiés ou non, émanant des établissements d'enseignement et de recherche français ou étrangers, des laboratoires publics ou privés. 


\title{
Visual Servoing/Tracking Using Central Catadioptric Images
}

\author{
João P. Barreto ${ }^{1}$, Frédérick Martin² ${ }^{2}$ and Radu Horaud ${ }^{2}$ \\ 1 Institute of Systems and Robotics \\ DEEC - University of Coimbra

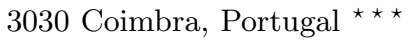 \\ 2 INRIA Rhône-Alpes \\ 655 Avenue de l'Europe \\ 38330 Montbonnot St. Martin, France
}

\begin{abstract}
Visual control of robot motion may benefit from enhanced camera field of view. With traditional cameras the available fields of view are only enough to view a region around the observed object (for eye-in-hand systems) or around the end-effector (for independent-eye systems). Central catadioptric systems have larger fields of view thus allowing the entire robot AND the surrounding objects to be imaged with a unique camera. Therefore, the whole robot's articulated mechanism can be observed and its joints can be tracked and controlled simultenously. This results in a new visual robot control concept where tracking and control are embedded together. Key to the understanding of both servoing and tracking is the central catadioptric Jacobian matrix linking the robot's joint velocities to image observations. In spite of a more complex projection matrix associated with catadioptric sensors, we study the catadioptric Jacobian matrix and we show that it does not introduce any additional singularity with respect to the traditional pinhole camera model. Experiments showing a rigid body being tracked with a catadioptric camera are described.
\end{abstract}

\section{Introduction}

Machine vision provides noncontact measurements of the world, extending the robot ability to operate in circumstances and environments which can not be accurately controlled. The approach of controlling motion using visual information is referred in the literature as visual servoing. Visual control of motion has been the object of intensive research in the last years. Several applications have been described for pose estimation [3], robot navigation [13] and positioning tasks of robotic manipulators [1,2].

Visual servoing applications can benefit from sensors providing large fields of view. The advantages of omnidirectional imaging in egomotion recovery from video were first discussed in [5]. Ambiguities and confusion between translation and rotation may arise whenever the translation direction lies outside the camera field of view. Panoramic sensors overcome this problem

\footnotetext{
$\star \star \star$ The work was partially supported by Foundation Calouste de Gulbenkian
} 
making the uncertainty of egomotion estimation independent of the direction of motion. More recently Aloimonos et al. proposed a spherical eye built with six cameras specifically designed for egomotion recovery [10]. Enhanced fields of view can also be advantageous for positioning tasks of robotic manipulators. The approaches to this problem are traditionally classified in two groups: position based and image based visual servoing [4]. In the former the control input is defined in the 3D task space. The pose of the target is estimated from image features based on the knowledge of a geometric model of the object and the camera calibration [1]. With only one camera there are ambiguities and singularities in pose estimation and the target can get out of the field of view during the tracking. In [3] a multiple camera approach is used to cope with these difficulties. Panoramic imaging can also overcome the refered problems avoiding multiple view geometry and calibration of several cameras.

One effective way to enhance the field of view of a camera is to use mirrors. The general approach of combining mirrors with conventional imaging systems is referred to as catadioptric image formation. In [6], Baker and Nayar derive the entire class of catadioptric systems with an unique viewpoint. Central catadioptric systems can be highly advantageous for many applications because they combine two important features: a single projection center and a wide field of view. Applications of these sensors in visual servoing, mainly for robot navigation purposes, appear in the literature [10,13]. However a global theory for visual control of motion using central catadioptric images has never been proposed.

This work introduces the Jacobian matrix $\mathbf{J}$ for a generic central catadioptric system. Matrix $\mathbf{J}$ is derived from the central catadioptric mapping function presented in [8]. According to this unifying theory, central catadioptric imaging can be modeled by a generic function $\mathbf{f}_{\mathbf{i}}$, with the type of sensor and shape of the mirror described by a parameter $\xi$. For the particular case of a conventional perspective camera the parameter $\xi$ is null. Thus, by assuming $\xi=0$, the general Jacobian matrix $\mathbf{J}_{\mathbf{g}}$ becomes the well known interaction matrix $\mathbf{J}_{\mathbf{p}}$ introduced the first time in [2]. Moreover it is shown that the derived Jacobian matrix can be decomposed in the product of two matrices $\mathbf{J}_{\mathbf{c}}$ and $\mathbf{J}_{\mathbf{p}}\left(\mathbf{J}_{\mathbf{g}}=\mathbf{J}_{\mathbf{c}} \cdot \mathbf{J}_{\mathbf{p}}\right)$. $\mathbf{J}_{\mathbf{c}}$ is a $2 \times 2$ matrix that is always invertible which proves that the general catadioptric Jacobian $\mathbf{J}_{\mathbf{g}}$ has exactly the same singularities as the standard perspective Jacobian $\mathbf{J}_{\mathbf{p}}[11,12]$.

Experiments on iterative pose estimation from points in the catadioptric image are performed. The singularities of $\mathbf{J}_{\mathbf{g}}$ and the stability and convergence of image based visual servoing from catadioptric images are discussed. Point-to-contour tracking [3] on omnidireccional images is used to estimate the rigid displacement of objects. The application of the derived framework to control the position of a robotic arm is also discussed. 


\section{Modelling Central Catadioptric Image Formation}

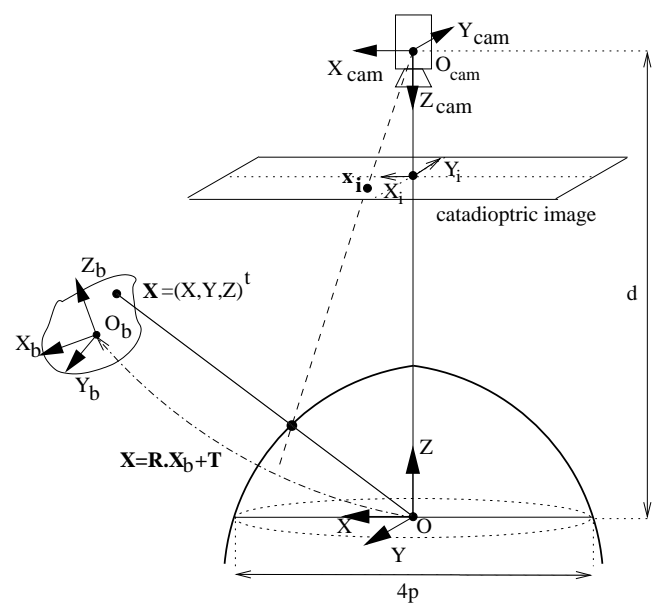

Fig. 1. Central catadioptric projection of a rigid body

A catadioptric realization of omnidirectional vision combines reflective surfaces and lenses. In [5], Baker et al. derive the entire class of catadioptric systems verifying the fixed viewpoint constraint. The fixed viewpoint constraint is a requirement ensuring that the visual sensor only measures the intensity of light passing through a single point in 3D space. An unique projection center is a necessary condition for the generation of geometrically correct perspective images [5], and for the existance of epipolar geometry inherent to the moving sensor and independent of the scene structure [7]. A central catadioptric system can be built by combining a parabolic mirror with an orthographic camera or an hyperbolic, elliptical or planar mirror with a perspective camera.

\begin{tabular}{|l|c|c|c|}
\hline & Mirror Surface & $\xi$ & $\psi$ \\
\hline Parabolic & $\sqrt{X^{2}+Y^{2}+Z^{2}}=2 p-Z$ & 1 & $1+2 p$ \\
\hline Hyperbolic & $\frac{\left(Z-\frac{d}{2}\right)^{2}}{\left(\frac{1}{2}\left(\sqrt{d^{2}+4 p^{2}}-2 p\right)\right)^{2}}-\frac{X^{2}+Y^{2}}{p\left(\sqrt{d^{2}+4 p^{2}}-2 p\right)}=1$ & $\frac{d}{\sqrt{d^{2}+4 p^{2}}}$ & $\frac{d+2 p}{\sqrt{d^{2}+4 p^{2}}}$ \\
\hline Elliptical & $\frac{\left(Z-\frac{d}{2}\right)^{2}}{\left(\frac{1}{2}\left(\sqrt{d^{2}+4 p^{2}}+2 p\right)\right)^{2}}+\frac{X^{2}+Y^{2}}{p\left(\sqrt{d^{2}+4 p^{2}}+2 p\right)}=1$ & $\frac{d}{\sqrt{d^{2}+4 p^{2}}}$ & $\frac{d-2 p}{\sqrt{d^{2}+4 p^{2}}}$ \\
\hline Planar & $Z=\frac{d}{2}$ & 0 & 1 \\
\hline
\end{tabular}

Table 1. Column 1: Reflective surfaces for the different cases of central panoramic imaging. Column 2 and 3: Parameters $\xi$ and $\psi$ of the general central catadioptric model 
Fig.1 is a scheme of the catadioptric system combining an hyperbolic reflective surface with a perspective camera. Consider the coordinate systems $\Re$ and $\Re_{\text {cam }}$ associated respectively with the mirror and the perspective camera. The hyperbola axis is coincident with the Z-axis of $\Re$, and its foci are coincident with $\mathbf{O}$ and $\mathbf{O}_{\text {cam }}$ (the origins of $\Re$ and $\Re_{\text {cam }}$ ). The latus rectum of the hyperbolic surface is $4 p$ and the distance between the foci is $d$. Light rays incident with $\mathbf{O}$ (the inner focal point) are reflected into rays incident with $\mathbf{O}_{\text {cam }}$ (the outer focal point). If the projection center of the perspective camera is coincident with $\mathbf{O}_{\text {cam }}$ the the captured light rays go originally through the inner focus of the hyperbolic surface. The effective viewpoint of the grabbed image is $\mathbf{O}$ and is unique. Elliptical catadioptric images are obtained combining an elliptical mirror with a perspective camera in a similar way. In the parabolic situation a parabolic mirror is placed such that its axis is the Z-axis, and its unique finite real focus is coincident with $\mathbf{O}$. Light rays incident with $\mathbf{O}$ are reflected into rays parallel with the Z-axis which are captured by an orthographic camera with image plane perpendicular to the $\mathbf{Z}$-axis. The effective viewpoint is in $\mathbf{O}$ and is unique. A catadioptric system made up of a perspective camera steering a planar mirror also verifies the fixed viewpoint constraint. The effective projection center is behind the mirror in the perpendicular line passing through camera center. Its distance to the camera center is twice the distance between the planar mirror and the camera. Tab. 1 shows the equations of the different reflective surfaces.

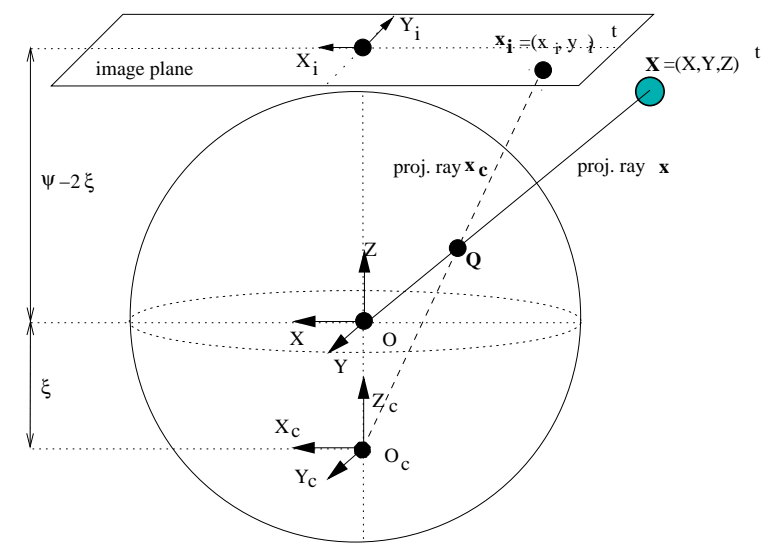

Fig. 2. Modelling central catadioptric image formation

In [8] Geyer and Daniilidis introduce an unifying theory for central catadioptric systems. Assume that a point with $3 \mathrm{D}$ coordinates $\mathbf{X}=(X, Y, Z)^{t}$ is projected in $\mathbf{x}_{\mathbf{i}}=\left(x_{i}, y_{i}\right)^{t}$ in the catadioptric image plane (Fig. 1). It can be shown that central panoramic projection is isomorphic to a projective map- 
ping from a sphere to a plane. Consider the scheme of Fig. 2 with the unitary sphere centered in the effective viewpoint $\mathbf{O}$, the point $\mathbf{O}_{\mathbf{c}}$ with coordinates $(0,0,-\xi)^{t}$ and the plane $Z=\psi-2 \xi$ orthogonal to the $\mathrm{Z}$ axis. Both $\xi$ and $\psi$ are function of mirror parameters $d$ and $p$ (Tab. 1). The projective ray $\mathbf{x}$ going through $\mathbf{X}$ intersects the spherical surface in $\mathbf{Q}=\left(\frac{X}{\rho}, \frac{Y}{\rho}, \frac{Z}{\rho}\right)^{t}$ with $\rho=\sqrt{X^{2}+Y^{2}+Z^{2}}$. A second projective ray $\mathbf{x}_{\mathbf{c}}$ can be defined by joining the intersection point $\mathbf{Q}$ with $\mathbf{O}_{\mathbf{c}}$. The intersection $\mathbf{x}_{\mathbf{i}}$ of the projective ray $\mathbf{x}_{\mathbf{c}}$ with the plane $Z=\psi-2 \xi$ is the catadioptric image of the original point $\mathbf{X}$. Central catadioptric imaging can be modeled by projecting the scene in the sphere surface and then re-projecting these points in the image plane from a novel projection center $\mathbf{O}_{\mathbf{c}}$. If the reflective surface is parabolic then $\xi=1$ and the re-projection is a stereographic projection. For the hyperbolic and elliptical mirror the re-projection center $\mathbf{O}_{\mathbf{c}}$ is inside the sphere in the negative $\mathrm{Z}$ axis. The planar mirror is a degenerate case of central catadioptric imaging with $\xi=0$ and $\mathbf{O}_{\mathbf{c}}$ coincident with the effective viewpoint $\mathbf{O}$. Notice that a catadioptric sensor with a planar mirror is equivalent to a conventional perspective camera with a sign inversion in the $Y$ axis.

$$
\boldsymbol{f}_{\boldsymbol{i}}(\boldsymbol{X})=\left(\frac{f_{x}(\psi-\xi) X}{Z+\xi \sqrt{X^{2}+Y^{2}+Z^{2}}}-c_{x},-\frac{f_{y}(\psi-\xi) Y}{Z+\xi \sqrt{X^{2}+Y^{2}+Z^{2}}}-c_{y}\right)^{t}(1)
$$

The catadioptric image is acquired by a camera steering the reflective surface. Assume that that the $\mathrm{X}$ and $\mathrm{Y}$ camera focal lengths are respectively

$f_{x}$ and $f_{y}$ and $\mathbf{C}=\left(c_{x}, c_{y}\right)^{t}$ is the principal point. Equation 1 provides function $\mathbf{f}_{\mathbf{i}}$ which maps points in the scene in the catadioptric image plane $\left(\mathbf{x}_{\mathbf{i}}=\mathbf{f}_{\mathbf{i}}(\mathbf{X})\right)$. Any central catadioptric system with $\xi \neq 0$ can be easily calibrated from the image of three lines [9]. If the sensor calibration is known, function $\mathbf{f}_{\mathbf{i}}$ can be simplified by making $f_{x}(\psi-\xi)=f_{y}(\psi-\xi)=1$ and $c_{x}=c_{y}=0$. We will assume without loss of generality that the mapping function is given by equation 2 :

$$
\boldsymbol{f}_{\boldsymbol{i}}(\boldsymbol{X})=\left(\frac{X}{Z+\xi \sqrt{X^{2}+Y^{2}+Z^{2}}},-\frac{Y}{Z+\xi \sqrt{X^{2}+Y^{2}+Z^{2}}}\right)^{t}
$$

\section{Tracking and control}

Fig. 1 depicts a moving rigid object observed by a central catadioptric sensor. The referential frame $\Re_{\mathbf{b}}$ is attached to the moving body, $\mathbf{R}$ is the rotation matrix between $\Re_{\mathbf{b}}$ and $\Re$ and $\mathbf{T}$ is the position of $\mathbf{O}_{\mathbf{b}}$ in sensor coordinates. Our goal is to estimate the pose of the rigid body knowing the coordinates $\left\{\mathbf{X}_{b}^{1}, \mathbf{X}_{b}^{2}, \ldots, \mathbf{X}_{b}^{n}\right\}$ of a set of 'n' object points.

Let $\mathbf{X}_{\mathbf{b}}$ be a generic point of the object model. If the pose $(\mathbf{R}, \mathbf{T})$ is known then the point 3D position in sensor coordinates is $\mathbf{X}=\mathbf{R} \mathbf{X}_{\mathbf{b}}+\mathbf{T}$. From equation 2 it comes that point $\mathbf{X}$ is projected in $\mathbf{x}_{\mathbf{i}}=\mathbf{f}_{\mathbf{i}}(\mathbf{X})$ in the catadioptric image plane. Object rigid motion implies a change in pose that 


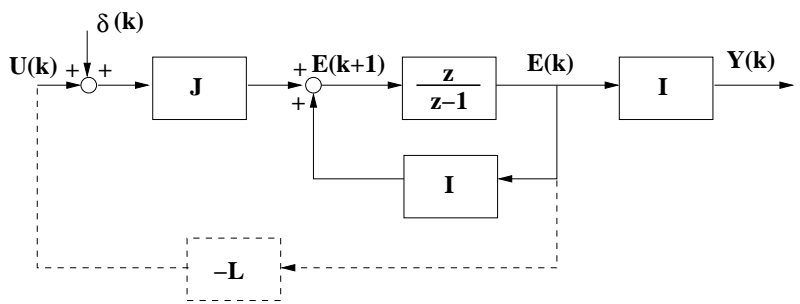

Fig. 3. Iterative pose estimation is a regulation control problem. $\mathbf{I}$ is the $2 n \times 2 n$ identity matrix. The dashed line corresponds to the feedback loop.

can be described by a kinematic screw $\delta=(\omega, \mathbf{v})^{\mathbf{t}}$. Consider the $3 \times 6$ matrix $\mathbf{J}_{\mathbf{m}}=[\tilde{\mathbf{X}} \mid \mathbf{I}]$ where $\tilde{\mathbf{X}}$ is the skew-symetric matrix of $\mathbf{X}$ and $\mathbf{I}$ is the $3 \times 3$ identity matrix. The $3 \mathrm{D}$ velocity of point $\mathbf{X}$ due to object rigid motion is $\dot{\mathbf{X}}=\mathbf{J}_{\mathbf{m}} \delta$. Moreover if $\mathbf{J}_{\mathbf{i}}$ is the Jacobian matrix of function $\mathbf{f}_{\mathbf{i}}$ (equation 2) and $\mathbf{J}_{\mathbf{g}}=\mathbf{J}_{\mathbf{i}} \mathbf{J}_{\mathbf{m}}$ then the corresponding velocity in the catadioptric image plane is $\dot{\mathbf{x}}_{\mathbf{i}}=\mathbf{J}_{\mathbf{g}} \delta$.

$$
E=\left[\begin{array}{c}
x_{i}^{1}-\hat{x}_{i}^{1} \\
x_{i}^{2}-\hat{x}_{i}^{2} \\
\vdots \\
x_{i}^{n}-\hat{x}_{i}^{n}
\end{array}\right] \approx\left[\begin{array}{c}
J_{g}^{1} \\
J_{g}^{2} \\
\vdots \\
J_{g}^{n}
\end{array}\right] \hat{\delta}=J \hat{\delta}
$$

Let $\hat{\mathbf{s}}=\left\{\hat{\mathbf{x}}_{i}^{1}, \hat{\mathbf{x}}_{i}^{2}, \ldots, \hat{\mathbf{x}}_{i}^{n}\right\}$ be the set of model points projected in the image accordingly to a certain pose estimation $(\hat{\mathbf{R}}, \hat{\mathbf{T}})$, and $\mathbf{s}=\left\{\mathbf{x}_{i}^{1}, \mathbf{x}_{i}^{2}, \ldots, \mathbf{x}_{i}^{n}\right\}$ the real positions of those points. Vector $\mathbf{E}$ is defined as $\mathbf{E}=\mathbf{s}-\hat{\mathbf{s}}$ and depends on the pose estimation error described by the kinematic screw $\hat{\delta}$. From the above discussion it comes that $\left(\mathbf{x}_{\mathbf{i}}^{\mathbf{j}}-\hat{\mathbf{x}}_{\mathbf{i}}^{\mathbf{j}}\right) \approx \mathbf{J}_{\mathbf{g}}^{\mathbf{j}} \hat{\delta}$ with $j=1,2, \ldots, n$ and $\mathbf{J}_{\mathbf{g}}^{\mathbf{j}}$ the Jacobian matrix $\mathbf{J}_{\mathbf{g}}$ evaluated on the $j^{\text {th }}$ model point. Equation 3 establishes the relationship between the measured image error $\mathbf{E}$ and the error $\hat{\delta}$ on the pose estimation of the rigid body. $\mathbf{J}$ is a $2 n \times 6$ matrix comprised by the Jacobian matrix $\mathbf{J}_{\mathbf{g}}$ evaluated in the 'n' points of the object model. The objective is to update the pose estimation such that the image of the model becomes coincident with the object image and the measured error vector $\mathbf{E}$ converges to zero.

$$
\left\{\begin{array}{l}
E(k+1)=I E(k)+J \delta(k)+J U(k) \\
Y(k)=E(k)
\end{array}\right.
$$

The problem stated in the previous paragraph can be formulated as a regulation control problem. Consider the system whose block diagram is depicted in Fig. 3. The state vector is the error $\mathbf{E}(\mathbf{k})$ measured in the catadioptric image, the system input matrix is $\mathbf{J}$ (equation 3), and the system output is $\mathbf{Y}(\mathbf{k})$ which must be zero. Accordingly to the system state-space equation 4 
the pose change $\delta$ acts as a perturbation disturbing the output $\mathbf{Y}(\mathbf{k})$. The purpose is to find a state feedback controller $\mathbf{L}$ such that if $\mathbf{U}(\mathbf{k})=-\mathbf{L E}(\mathbf{k})$ then the disturbance is rejected and the system state converges to zero.

$$
\begin{aligned}
& L=\left(J^{t} J\right)^{-1} J^{t} \\
& \left\{\begin{array}{l}
E(k+1)=\left(I-J\left(J^{t} J\right)^{-1} J^{t}\right) E(k)+J \delta(k) \\
\boldsymbol{Y}(k)=E(k)
\end{array}\right.
\end{aligned}
$$

The least squares solution of equation 3 is $\hat{\delta}(\mathbf{k})=\left(\mathbf{J}^{\mathbf{t}} \mathbf{J}\right)^{-\mathbf{1}} \mathbf{J}^{\mathbf{t}} \mathbf{E}(\mathbf{k}) . \hat{\delta}(\mathbf{k})$ is an estimate of the pose error associated with the measured image error $\mathbf{E}(\mathbf{k})$. System regulation can be achieved by making $\mathbf{U}(\mathbf{k})=\hat{\delta}(\mathbf{k})$ (equation 5). Equation 6 provides the state space model of the final closed loop system. System stability and transient response depend on the eigenvalues of the matrix $\left(\mathbf{I}-\mathbf{J}\left(\mathbf{J}^{\mathbf{t}} \mathbf{J}\right)^{-\mathbf{1}} \mathbf{J}^{\mathbf{t}}\right)$. However it is important to remind that the state transition matrix is a function of rigid body position which changes along time. Moreover the controller of equation 5 is only realizable when $\left(\mathbf{J}^{\mathbf{t}} \mathbf{J}\right)$ is non singular. Whenever matrix $\left(\mathbf{J}^{\mathbf{t}} \mathbf{J}\right)$ is not invertible we are in presence of a singularity.

\section{The Jacobian Matrix for General Central Catadioptric Projection}

To design the controller of equation 5 we need to obtain matrix $\mathbf{J}$ depending on the Jacobian matrix $\mathbf{J}_{\mathbf{g}}$ which is evaluated on the 'n' points of the object model (equation 3).

$$
\boldsymbol{J}_{\boldsymbol{i}}=\frac{1}{\rho(Z+\xi \rho)^{2}}\left[\begin{array}{ccc}
\rho Z+\xi\left(Y^{2}+Z^{2}\right) & -\xi X Y & -X(\rho+\xi Z) \\
\xi X Y & -\left(\rho Z+\xi\left(X^{2}+Z^{2}\right)\right) & Y(\rho+\xi Z)
\end{array}\right](7)
$$

Consider the central catadioptric mapping function $\mathbf{f}_{\mathbf{i}}$ which maps $3 \mathrm{D}$ point coordinates $\mathbf{X}$ in image coordinates $\mathbf{x}_{\mathbf{i}}$. The corresponding Jacobian matrix $\mathbf{J}_{\mathbf{i}}$ is derived by diferentiating the function of equation 2 . The achieved result is presented on equation 7 where $\rho=\sqrt{X^{2}+Y^{2}+Z^{2}}$.

$$
\boldsymbol{J}_{\boldsymbol{g}}=\left[\begin{array}{cccc}
x_{i} y_{i} & \frac{\left(1+x_{i}^{2}\right) \Upsilon-y_{i}^{2} \xi}{\Upsilon+\xi} & y_{i} & \frac{1+x_{i}^{2}(1-\xi(\Upsilon+\xi))+y_{i}^{2}}{\rho(\Upsilon+\xi)} \\
\frac{\left(1+y_{i}^{2}\right) \Upsilon-x_{i}^{2} \xi}{\Upsilon+\xi} & x_{i} y_{i} & -x_{i} & -\frac{x_{i} y_{i} \xi}{\rho} \\
& & \frac{x_{i} y_{i} \xi}{\rho} & -\frac{x_{i} \Upsilon}{\rho} \\
& & \frac{1+x_{i}^{2}+y_{i}^{2}(1-\xi(\Upsilon+\xi))}{\rho(\Upsilon+\xi)}-\frac{y_{i} \Upsilon}{\rho}
\end{array}\right]
$$

Assume $\mathbf{J}_{\mathbf{i}}$ provided by equation 7 and $\mathbf{J}_{\mathbf{m}}=[\tilde{\mathbf{X}} \mid \mathbf{I}]$ with $\tilde{X}$ the skew symetric matrix associated with point coordinates $\mathbf{X}$. It was already shown that the Jacobian matrix $\mathbf{J}_{\mathbf{g}}$ can be computed as $\mathbf{J}_{\mathbf{g}}=\mathbf{J}_{\mathbf{i}} \mathbf{J}_{\mathbf{m}}$. Equation 8 presents 
the general central catadioptric Jacobian matrix as a function of image position $\mathbf{x}_{\mathbf{i}}$, point depth $\rho$ and sensor $\xi$ parameter $\left(\Upsilon=\sqrt{1+\left(x_{i}^{2}+y_{i}^{2}\right)\left(1-\xi^{2}\right)}\right)$. Notice that if $\xi=0$ then matrix $\mathbf{J}_{\mathbf{g}}$ becomes the well known Jacobian matrix $\mathbf{J}_{\mathbf{p}}$ introduced in [2] for conventional perspective cameras.

$$
\boldsymbol{J}_{\boldsymbol{i}}=\underbrace{\left[\begin{array}{cc}
\frac{Z\left(\rho Z+\xi\left(Y^{2}+Z^{2}\right)\right)}{\rho(Z+\xi \rho)^{2}} & \frac{\xi X Y Z}{\rho(Z+\xi \rho)^{2}} \\
\frac{\xi X Y Z}{\rho(Z+\xi \rho)^{2}} & \frac{Z\left(\rho Z+\xi\left(X^{2}+Z^{2}\right)\right)}{\rho(Z+\xi \rho)^{2}}
\end{array}\right]}_{\mathbf{J}_{\mathbf{c}}}\left[\begin{array}{ccc}
\frac{1}{Z} & 0 & -\frac{X}{Z^{2}} \\
0 & -\frac{1}{Z} & \frac{Y}{Z^{2}}
\end{array}\right]
$$

The Jacobian $\mathbf{J}_{\mathbf{i}}$ of the mapping function $\mathbf{f}_{\mathbf{i}}$ can be decomposed in the matrix product of equation 9 . $\mathbf{J}_{\mathbf{c}}$ is the $2 \times 2$ matrix depending on point coordinates $\mathbf{X}$ and on the mirror parameter $\xi$. If $\xi=0$ then $\mathbf{J}_{\mathbf{c}}$ is the identity matrix. The second matrix has dimension $2 \times 3$ and it is the Jacobian matrix of the perspective mapping function $\mathbf{f}_{\mathbf{i}}=(X / Z,-Y / Z)^{t}$ obtained making $\xi$ equal to zero in equation 2 . Thus the general catadioptric matrix $\mathbf{J}_{\mathbf{g}}$ can be written as $\mathbf{J}_{\mathbf{g}}=\mathbf{J}_{\mathbf{c}} \mathbf{J}_{\mathbf{p}}$ with $\mathbf{J}_{\mathbf{p}}$ the $2 \times 6$ Jacobian for the perspective camera situation. Moreover for $Z>0$ the square matrix $\mathbf{J}_{\mathbf{c}}$ is positive definite with eigenvalues $\left\{Z /(Z+\rho \xi) ;\left(Z^{2}(\rho+\xi Z)\right) /\left(\rho(Z+\xi \rho)^{2}\right)\right\}$.

$$
J=\underbrace{\left[\begin{array}{cccc}
J_{c}^{1} & \mathbf{0} & \ldots & \mathbf{0} \\
\mathbf{0} & \boldsymbol{J}_{c}^{2} & \ldots & \mathbf{0} \\
\vdots & \vdots & \ddots & \vdots \\
\mathbf{0} & 0 & \ldots & J_{c}^{n}
\end{array}\right]}_{\mathrm{C}} \underbrace{\left[\begin{array}{c}
J_{p}^{1} \\
J_{p}^{2} \\
\vdots \\
J_{p}^{n}
\end{array}\right]}_{\mathrm{P}}
$$

The controller of equation 5 is realizable if and only if $\mathbf{J}$ is a full rank matrix. J has dimension $2 n \times 6$ where ' $\mathrm{n}$ ' is the number of considered model points. Clearly the full rank constraint can not be verified with less than three points. Equation 10 is derived from equation 3 knowing that $\mathbf{J}_{\mathbf{g}}^{\mathbf{j}}=\mathbf{J}_{\mathbf{c}}^{\mathbf{j}} \mathbf{J}_{\mathbf{p}}^{\mathbf{j}}$. Matrix $\mathbf{J}$ is the product of a $2 n \times 2 n$ square matrix $\mathbf{C}$ with a matrix $\mathbf{P}$ with dimension $2 n \times 6$. It was shown that $\mathbf{J}_{\mathbf{c}}^{\mathbf{j}}$ is positive definite for $j=1,2, \ldots, n$ and matrix $\mathbf{C}$ is always full rank. This poofs that $\mathbf{J}$ is rank deficient only when $\mathbf{P}$ is also rank deficient. The general central catadioptric situation does not present more singularities than the perspective case. These singularities were studied in $[12,3]$.

\section{$5 \quad$ Tracking experiments and Conclusions}

Based on the tracking method described above we implemented an object tracker. Since with catadioptric cameras straight lines map onto the image plane as quadrics, we devised a contour-to-point tracker along the lines described in [3]. The figures below show a rectangular object moving towards the camera and in a direction perpendicular to the camera. The advantage of 

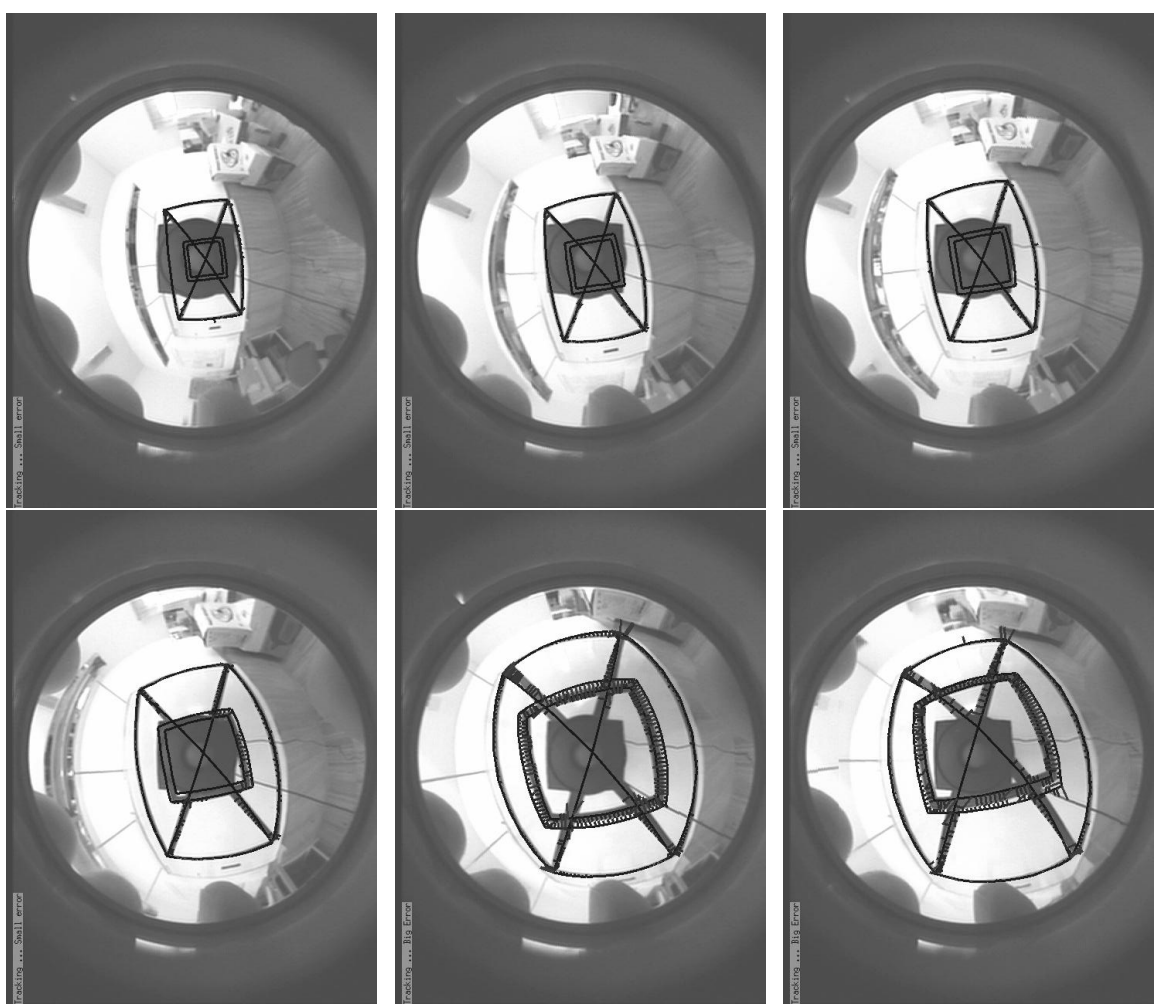

Fig. 4. A tracking sequence. The object translates along axis of camera

this method is that only points along contours are to be found in the image thus avoiding the tedious and unreliable process of fitting a quadric to a set of points.

The model based tracking of a rigid object can be exploited in many ways for visual servoing applications. The proposed approach is being used in robot navigation and cooperation [13]. The experimental setup consists in two mobile plataforms both equipped with central catadioptric cameras. A visual landmark, similar to the one depicted in the figures, is positioned in the room ceil. One robot is the leader with independent motion and the other is the slave. The objective is to control slave motion such that the relative position between the two plataforms is kept constant. To achieve this goal both robots use the omnidirectional vision to estimate their pose from the model based tracking of the landamark. A method to control the position of a robotic arm using a static catadioptric system is also being develoed. Typically, in visual servoing using a conventional perspective camera, the available field of view is only enough to image the region around the endeffector. The pose of the end-effector is estimated by visual feedback, and motion control is achieved using the manipulator jacobian known "a priori". 

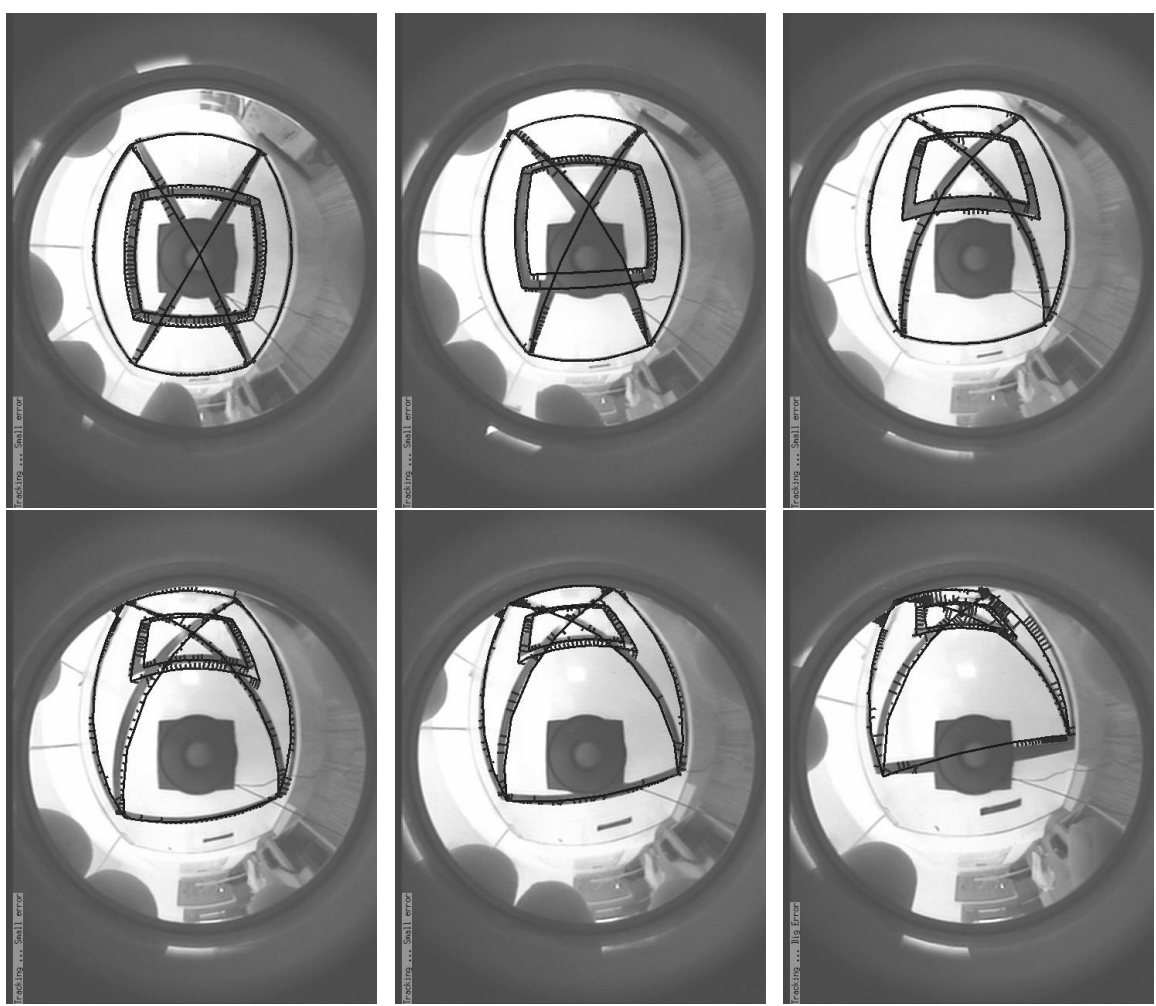

Fig. 5. A tracking sequence. The object translates in front of camera..

The success of this approach is highly dependent on the arm calibration. We use the wide field of view provided by the omnidirectional sensor to image the entire arm. The different manipulator links are tracked in the catadioptric image and the motion of each joint is estimated. This approach increases the robustness and accuracy of the visual servoing.

\section{References}

1. W. Wilson, C. Hulls, and G. Belles, "Relative end effector control using cartesian position-based visual servoing," IEEE Trans. on Robotics and Automation, vol. 12, no. 5, pp. 684-696, October 1996.

2. B. Espiau, F. Chaumette, and P. Rives, "A new approach to visual servoing in robotics," IEEE Trans. on Robotics and Automation, vol. 8, no. 3, pp. 313-326, June 1992.

3. F. Martin and R. Horaud, "Multiple Camera Tracking of Rigid Objects", Research report 4268 INRIA, Montbonnot, France, September 2001.

4. S. Hutchinson, G. Hager, and P. I. Corke, "A tutorial on visual servo control," IEEE Trans. on Robotics and Automation, vol. 12, no. 5, pp. 651-670, October 1996. 
5. J. Gluckman and S. Nayar, " Egomotion and Omnidirectional Cameras," ICCV98 - Proc. IEEE International Conference on Computer Vision, pp. 9991005, Bombay 1998.

6. S. Baker and S. Nayar, "A Theory of Catadioptric Image Formation," ICC V98 - Proc. IEEE International Conference on Computer Vision, pp. 35-42, Bombay 1998.

7. T. Svoboda, T. Pajdla and V. Hlavac, "Motion Estimation Using Central Panoramic Cameras," Proc. IEEE Conference on Intelligent Vehicles, Stugart Germany 1998.

8. C. Geyer and K. Daniilidis, "A Unifying Theory for Central Panoramic Systems and Pratical Implications," ECCV2000-Proc. European Conference on Computer Vision, pp. 445-461, Dublin 2000.

9. Joao P. Barreto and H. Araujo, "Geometric Properties of Central catadioptric Line Images," in Proc. of the European Conference on Computer Vision, Copenhag, Denmark, May 2002.

10. P. Baker, C. Fermuller, Y. Aloimonos and R. Pless, "A Spherical Eye From Multiple Cameras (Makes Better Models of the World)," in Proc. of the IEEE Int. Conf. on Computer Vision and Pattern Recognition, Kauai, Haway, USA, December 2001.

11. Francois Chaumette, "Potential Problems of Stability and Convergence in Image Based and Position Based Visual Servoing", The Confluence of Vision and Control, Lecture Notes in Control and Information Systems, Vol. 237, pp 66-78, Springer-Verlag, 1998.

12. H. Michel and P. Rives, "Singularities in the determination of the situation of a robot effector from the perspective view of 3 points", Research report 1850 INRIA, Sophia-Antipolis, France, February 1993.

13. A. Paulino and H. Araujo, "Multiple Robots in Geometric Formation: Control Structure and Sensing", in Int. Symposyum on Intelligent Robotic Systems, Reading, UK, 2000. 